\title{
EL AULA DE EDUCACIÓN FÍSICA Y EL ALUMNADO CON DISCAPACIDAD
}

\section{THE CLASSROOM OF PHYSICAL EDUCATION AND STUDENTS WITH DISABILITIES}

\author{
Macías García, D. ${ }^{1}$; González López, I. ${ }^{2}$ y López Urbán, A.I. ${ }^{3}$ \\ ${ }^{1}$ Doctor en Psicopedagogía. Junta de Andalucía. España. \\ david.macias.garcia.edu@juntadeandalucia.es \\ ${ }^{2}$ Doctor en Pedagogía. Facultad de Ciencias de la Educación. Universidad de Córdoba. España. \\ ignacio.gonzalez@uco.es \\ ${ }^{3}$ Licenciada en Pedagogía. Junta de Andalucía. España. anaycarmen89@hotmail.com
}

Código UNESCO: 5801 Teoría y Métodos Educativos

Clasificación Consejo de Europa: 5. Didáctica y metodología

Recibido el 12 de marzo de 2017

Aceptado el 3 de junio de 2017

Correspondencia:

Ignacio González López

ignacio.gonzalez@uco.es

\section{RESUMEN}

Este estudio versa sobre el proceso de construcción y validación de un instrumento estandarizado cuyo objetivo ha sido analizar la realidad educativa de los docentes de Educación Física en la etapa de primaria ante alumnado con discapacidad motora y derivar del mismo los elementos clave que justifiquen la construcción de un plan de formación permanente del profesorado en este ámbito. El proceso llevado a cabo pasó por llevar a cabo en primer lugar un juicio de expertos y expertas para posteriormente someter el borrador de aquí derivado a un pilotaje con un total de 57 docentes de Córdoba. El cuestionario definitivo goza de las suficientes garantías para ser aplicado, analizado e interpretado y ha permitido extraer como dimensiones formativas las siguientes: didáctica de la Educación Física, herramientas didáctico educativas, estrategias de trabajo en el aula, habilidades para el ejercicio de la docencia y recursos tecnológicos.

PALABRAS CLAVE: Educación Física, discapacidad motora, formación permanente, escala de valoración 


\section{ABSTRACT}

This study focuses on the process of construction and validation of a standardized instrument whose aim was to analyze the educational reality of teachers of physical education at the primary stage before pupils with motor disabilities and derive the same key elements that would justify the construction of a permanent training of teachers in this area plan. The process carried out step to carry out first a judgement of experts for subsequently derived here draft will undergo a pilot with a total of 57 teachers from Córdoba. The final questionnaire enjoys sufficient safeguards to be applied, analysed and interpreted and allowed extract as dimensions training as follows: Didactics of physical education, educational teaching tools and strategies of work in the classroom, skills for the exercise of the teachers and technological resources.

KEY WORDS: Physical education, physics disability, permanent training, rating scale

\section{INTRODUCCIÓN}

La diversidad es un concepto polivalente en una doble perspectiva educativa y social que surge por la necesidad de atender a las necesidades de cada alumno y alumna, fruto de la heterogeneidad (Muntaner, 2000). Esta bidimensionalidad tiene su respuesta en el modelo derivado de la escuela inclusiva, que busca atender a las necesidades del aprendizaje de todas y todos los niños, jóvenes y adultos, teniendo especial atención en aquellos que sean más vulnerables a la marginalidad y la exclusión social (Echeita y Ainscow, 2011).

Dentro de esta vulnerabilidad se encuentra el alumnado con discapacidad, característica que ha derivado de un paradigma deficitario individual que considera la discapacidad como un problema de la persona causado por su condición de salud (trauma o enfermedad) y que necesita cuidados médicos, a un paradigma competencial basado en un modelo social, centrado en el entorno del individuo (Egea y Sarabia, 2014).

Bajo esta segunda perspectiva, Ríos y Martínez (2004) y Beltrán (2008) explican que dentro del alumnado con discapacidad, la denominada discapacidad motora es una alteración del sistema locomotor que afecta fundamentalmente a la ejecución de movimientos. Díaz (2010, p. 2) entiende la discapacidad motora como una «deficiencia que abarca todas las alteraciones $o$ deficiencias orgánicas del aparato locomotor o de su funcionamiento que afectan al sistema óseo, articulaciones, nervios y/o músculos. Las personas afectadas por ellas presentan una clara desventaja en su manipulación, pudiendo integrar dos o más de estas. Lo importante del proceso de intervención no debe centrarse en el déficit que presenta el alumno, sino en las posibilidades de aprendizaje y las potencialidades educativas que este pueda desarrollanı. La Junta de Andalucía (2015) estableció a través de unas instrucciones normativas la clasificación atribuida a este tipo de discapacidades y que son las siguientes: lesiones cerebrales, lesiones medulares, trastornos neuromusculares y lesiones del sistema osteoraticular. 
Dentro de las diferentes áreas del currículo educativo nos encontramos con la Educación Física, cuya finalidad es satisfacer las necesidades de movimiento de las personas desarrollando simultáneamente sus capacidades físicas y motrices, instruyendo sobre el funcionamiento del propio cuerpo y sobre los efectos del ejercicio físico, analizando las relaciones entre salud y actividad motriz. Tomando como referencia la normativa vigente en Andalucía (Decreto 97/2015), las destrezas, habilidades y capacidades de este área se organizan en torno a cuatro bloques de contenido: el cuerpo y sus habilidades perceptivo motrices, la Educación Física como favorecedora de salud, la expresión corporal: expresión y creación artística motriz y el juego y deporte escolar. Estos contenidos se trabajan para conseguir el desarrollo de las competencias clave (Muñoz, 2014). Se trata ésta de un área de acción y reflexión donde las actividades deben ser valoradas desde el proceso de la actividad motriz en sí misma y nunca desde los resultados (Velázquez, Aranda y Pastor, 2014) y se procurará que las propuestas integren distintos aspectos con el consiguiente enriquecimiento de las experiencias de aula (Pantoja y Montijano, 2014): colaboración, juego limpio, autonomía personal, autoconocimiento, superación de dificultades, calidad de vida, vida saludable, hábitos alimenticios y de higiene y resolución de conflictos. Por su parte, la evaluación debe girar en torno a su planteamiento más formativo basada en los procesos que quedan definidos en los objetivos del área. Se evalúa para enseñar y aprender, considerando el esfuerzo y el trabajo realizado frente al resultado, es decir, se convierte en fundamental el respeto a los ritmos de trabajo (Arnaiz, De Haro y Guijarro, 2015). En definitiva, la Educación Física es una de las áreas donde se facilita el desarrollo integral, el proceso de maduración y la integración de todo alumnado al aula, ya que se centra en el trabajo colectivo, incentiva la participación, la resolución de problemas y la convivencia con el grupo-clase. Esta consideración del área entronca directamente con los preceptos de una escuela inclusiva, donde se debe posibilitar que todo el alumnado pueda desarrollar al máximo sus aprendizajes dentro de cada una de las áreas del currículo (Muntaner, 2010).

Ríos (2009) explica que cuando hablamos de una Educación Física inclusiva todo el alumnado comparte el mismo espacio, sin diferencias, reconsiderando la enseñanza y su organización con el apoyo pedagógico y social que sea necesario, y manteniendo las más altas expectativas para el aprendizaje de todas y todos. La inclusión presupone compartir con el grupo el proceso de aprendizaje y en este contexto la diversidad cohesiona al grupo y lo enriquece, ofreciendo más posibilidades de aprendizaje para todos y todas. No obstante, existen algunas condicionantes o barreras que pueden dificultar la consecución de la inclusión en este área (Ríos, 2009; Caus, Santos, Blasco y Vega, 2013; Penagos, 2013 y Jenaro et al., 2014): escasez de recursos económicos, elevada ratio, dificultades de accesibilidad física, infravaloración del área e inadecuada formación del profesorado.

Los análisis de Díaz (2010) demuestran que este grupo docente tiene inquietud por conocer y obtener recursos que le permitan atender a la diversidad de características, necesidades, pautas de actuación y comportamientos que presenta el alumnado con discapacidad. Desde estas premisas, el III Plan Andaluz de Formación Permanente del Profesorado (Orden de 31 de julio de 
2014) señala la importancia de formar al profesorado en el ámbito de la educación inclusiva. Este programa, construido desde la prolongada experiencia que ha desarrollado la red de formación en la comunidad autónoma, concreta las líneas estratégicas de actuación en materias de formación que sirven de soporte para la implantación de las líneas educativas que desde la Consejería de Educación se llevan a cabo. Dentro de la línea 1, "La formación del profesorado vinculada a la mejora de las prácticas educativas, el rendimiento y el éxito educativo de todo el alumnado», se aborda el eje "Escuela inclusiva para la igualdad y la equidad: atención a la diversidad, convivencia e igualdad», elemento a partir del cuál se ha generado este trabajo.

\section{OBJETIVOS}

La pretensión de este trabajo es diseñar un instrumento de recogida de información que permita descubrir, analizar y sistematizar la realidad que se produce en las clases de Educación Física en educación primaria con el alumnado con algún tipo de discapacidad física, estimando sus garantías científicas de fiabilidad y validez para que pueda ser aplicado de manera generaliza y proporcione los elementos clave que sirvan para el diseño de planes de formación que permitan optimizar la labor profesional de este grupo docente.

\section{MATERIAL Y MÉTODOS}

El estudio responde a una metodología de tipo exploratorio descriptivo al amparo de un diseño de investigación de carácter no experimental. Se ha trabajado con un total de 69 variables, concretadas y clasificadas en las siguientes dimensiones:

- Características del profesorado: esta primera dimensión recoge todos los aspectos relacionados con la información sociodemográfica y académica de los y las docentes para poder realizar una correcta descripción del grupo informante. Las variables que la configuran pueden dividirse en tres subdimensiones. La primera hace referencia a las características sociodemográficas del profesorado, la segunda está constituida por las actividades de formación que realiza y los motivos por las que acude a ellas $y$, en tercer lugar, se desea conocer si este profesorado ha trabajado en su aula con alumnado con algún tipo de discapacidad, conociendo cuál es la que predomina.

- Concepción de la discapacidad en educación primaria y en Educación Física: en esta dimensión englobamos todas las variables referentes al conocimiento de las diferentes discapacidades y sus características, así como a la percepción de los efectos que produce en el aula la presencia de alumnado con estas características (Ríos, 2007). Se recoge información sobre la integración del alumnado con discapacidad dentro del aula y cómo se siente tanto el estudiante con discapacidad como el resto de estudiantes, es decir, sobre los aspectos positivos y los aspectos a mejorar en el trabajo de aula con alumnado con algún tipo de discapacidad. 
- Planificación didáctica y diseño curricular: en esta dimensión recogemos una serie de variables que hacen referencia a los elementos curriculares de la programación didáctica docente, a las medidas para atender a la diversidad a adoptar y al cómo llevarlas a cabo y a las y los profesionales que pueden participar de manera coordinada para mejorar dicha programación.

- Necesidades formativas: en las variables que aparecen en esta dimensión se presentan una serie de elementos que pretenden detectar cuáles son las necesidades formativas más relevantes del maestro o maestra de Educación Física. Algunos de ellos hacen referencia a las instituciones que proporcionan formación docente y a su calidad, mientras que otros hacen alusión al tipo de formación que requiere el o la docente para dar respuesta en las clases de Educación Física a las demandas del alumnado con discapacidad física.

- Demandas formativas: en último lugar, es relevante recabar información sobre estas demandas, intentado conocer cuáles son las dificultades a las que se enfrenta el docente en su práctica diaria y a partir de estos resultados crear un plan de trabajo formativo lo más adaptado posible a la realidad vigente (Junta de Andalucía, 2014).

Al plantearnos este estudio consideramos que la técnica de la encuesta, y dentro de los instrumentos existentes el cuestionario, era el instrumento más idóneo para poder ser respondido por las personas que componen la muestra de estudio (profesorado de educación primaria, especialista en Educación Física, de Córdoba capital y provincia). Igualmente, lo utilizamos porque era la forma más rápida y viable para que los y las participantes respondiesen al objetivo planteado. Se consideró diseñar un instrumento ad hoc debido a que la literatura científica no aportó ningún instrumento válido para este estudio. En su elaboración se han seguido los siguientes pasos:

- Paso 1. Elaboración provisional del cuestionario. Se diseñó un primer modelo de cuestionario a partir del objetivo perseguido. Se construyó a partir de una escala de medición tipo Likert compuesto por un conjunto de ítems presentados en forma de afirmaciones o juicios ante los cuales se pide la reacción de los y las participantes. La escala de medición abarca desde el 1 hasta el 5, siendo 1 el grado mínimo de acuerdo con el ítem planteado y 5 el grado máximo. El número inicial de preguntas ascendió a 69 agrupadas en las cinco dimensiones de estudio descritas anteriormente (ver tabla 1). 
Tabla 1. Descripción del cuestionario inicial dirigido al profesorado de Educación Física

\begin{tabular}{|c|c|c|c|}
\hline \multirow[b]{2}{*}{ Dimensiones } & \multirow{2}{*}{$\begin{array}{l}\mathrm{N}^{\circ} \mathrm{de} \\
\text { ítems }\end{array}$} & \multicolumn{2}{|l|}{$\begin{array}{l}\text { Tipología de ítems } \\
\end{array}$} \\
\hline & & Tipología & $\mathbf{N}$ \\
\hline \multirow{4}{*}{ - Características del profesorado } & \multirow{4}{*}{12} & Cerradas - dicotómicas & 4 \\
\hline & & Cerradas - politómicas & 1 \\
\hline & & Semicerradas & 1 \\
\hline & & Abiertas & 6 \\
\hline $\begin{array}{l}\text { - Concepción de la discapacidad en } \\
\text { educación primaria y Educación Física }\end{array}$ & 8 & $\begin{array}{l}\text { Cerradas - valoración } \\
\text { escalar }\end{array}$ & 8 \\
\hline $\begin{array}{l}\text { Planificación didáctica y diseño } \\
\text { curricular }\end{array}$ & 18 & $\begin{array}{l}\text { Cerradas - valoración } \\
\text { escalas }\end{array}$ & 18 \\
\hline - Necesidades formativas & 15 & $\begin{array}{l}\text { Cerradas - valoración } \\
\text { escalar }\end{array}$ & 15 \\
\hline - Demandas formativas & 16 & $\begin{array}{l}\text { Cerradas - valoración } \\
\text { escalar }\end{array}$ & 16 \\
\hline Total & 69 & & 69 \\
\hline
\end{tabular}

- Paso 2. Validación del cuestionario. Se llevaron a cabo dos procedimientos complementarios para dotar de garantías científicas al instrumento. En un primer momento y con el objeto de realizar una validez de contenido del instrumento a través de una tabla de especificaciones en la que se valore la pertinencia y la claridad de los elementos que lo definen, se presentó el cuestionario a doce profesores y profesoras de distintas universidades españolas (Córdoba, Sevilla e Islas Baleares) especializados en el ámbito objeto de esta investigación, a un maestro especialista de Educación Física con numerosas publicaciones en el área, a una maestra de educación especial con una dilatada experiencia en el trabajo con alumnado con discapacidad física, así como a dos asesores del departamento de formación de educación artística y corporal de dos centros de formación del profesorado de la provincia de Córdoba. Con este juicio de especialistas pretendimos alcanzar varios objetivos: a) corroborar la pertinencia de las respuestas dadas por el profesorado; b) poner de manifiesto el nivel de comprensión de la terminología presentada en el cuestionario; y c) observar las dificultades de aplicación del cuestionario, así como el tiempo de duración del mismo. En un segundo momento se procedió a realizar una aplicación experimental del cuestionario resultante a un grupo piloto para sondear las posibles respuestas y comprobar la consistencia interna, el poder de discriminación de los ítems y la validez de constructo. El grupo estuvo configurado por 56 docentes, la mayoría hombres $(70,9 \%)$, con una edad media de 37,55 años $(s=6,388)$ y una experiencia docente de 11,49 años $(s=5,791)$.

- Paso 3. Elaboración definitiva del cuestionario. Incorporadas las aportaciones del grupo de expertos y expertas así como los resultados del grupo piloto, el cuestionario definitivo se encuentra estructurado en cinco dimensiones que engloban 65 ítems, de los cuales 59 son preguntas cerradas y 6 son cuestiones abiertas. 


\section{RESULTADOS}

Se presentan a continuación los resultados obtenidos en las diferentes fases de validacion del cuestionario, así como los elementos clave que, desde la perspectiva del profesorado de Educación Física, servirán de base para la elaboración de un plan de formación docente para la atención educativa del alumnado con discapacidad física.

El primer procedimiento empleado fue un juicio de expertos y expertas (Ruiz, 2002), debido a que más allá de ser la validez instrumental expresada de modo cuantitativo mediante un índice o coeficiente, se estima su aproximación mediante un juicio generalmente subjetivo o intersubjetivo de expertos en la materia objeto de evaluación. El proceso consistió en proporcionar a las once personas expertas ya mencionadas un protocolo de evaluación consistente en la valoración de cada uno de los elementos redactados expresando una puntuación numérica de 1 a 5 (el 1 indica mínima valoración y el 5 máxima valoración), en función de su pertinencia (correspondencia entre el contenido del ítem y la dimensión para la cual va a ser utilizado) y su claridad (grado en que el ítem está redactado de forma clara y precisa, facilitando su comprensión por los sujetos encuestados). Además, en un apartado denominado "Comentarios / Formulación alternativa» asociado a cada una de las dimensiones a evaluar, las expertas y expertos pudieron realizar sus aportaciones y sugerencias al respecto y plantear modos alternativos de formulación de aquellos indicadores que consideraron inadecuados por su falta de claridad o pertinencia. Por último, pedimos que nos aportaran una definición teórica de cada una de estas dimensiones de estudio y su idoneidad en el modelo presentado.

Al acercarse a los resultados presentes en la tabla 2, pertenecientes a la dimensión «Concepción de la discapacidad en Educación Primaria y Educación Física», podemos decir que los ocho elementos propuestos son considerados pertinentes, es decir, que existe una correspondencia entre el contenido de los elementos y la dimensión bajo la que se amparan; y claros, lo que indica que están redactados de forma precisa, siendo comprensibles para las personas que son encuestadas. 
Tabla 2. Pertinencia y claridad de la dimensión «Concepción de la discapacidad en Educación Primaria y Educación Física»

\begin{tabular}{|c|c|c|}
\hline ELEMENTOS & $\mathbf{P}$ & C \\
\hline $\begin{array}{l}\text { 1. Conozco la clasificación de las diferentes discapacidades } \\
\text { motoras. }\end{array}$ & 4,00 & 4,27 \\
\hline $\begin{array}{l}\text { 2. Tengo claro lo que significa que un alumno tenga limitaciones } \\
\text { en la movilidad. }\end{array}$ & 4,36 & 4,09 \\
\hline $\begin{array}{l}\text { Considero complicado mantener unas buenas pautas de } \\
\text { clase en el área de Educación Física con alumnado con } \\
\text { limitaciones en la movilidad. }\end{array}$ & 4,10 & 4,00 \\
\hline $\begin{array}{l}\text { 4. El alumnado con limitaciones en la movilidad no puede llevar } \\
\text { a cabo en igualdad de condiciones la clase de Educación } \\
\text { Física como el alumnado que no posee limitaciones en la } \\
\text { movilidad }\end{array}$ & 4,40 & 4,80 \\
\hline $\begin{array}{l}\text { 5. La atención al alumnado con discapacidad es tarea del } \\
\text { especialista de pedagogía terapéutica. }\end{array}$ & 4,00 & 4,30 \\
\hline $\begin{array}{l}\text { 6. En las clases de Educación Física el alumnado con } \\
\text { limitaciones en la movilidad se siente diferente al alumnado } \\
\text { sin limitaciones en la movilidad. }\end{array}$ & 4,09 & 4,50 \\
\hline $\begin{array}{l}\text { 7. Considero que la diversidad del alumnado es enriquecedora } \\
\text { para todo el grupo. }\end{array}$ & 4,50 & 4,60 \\
\hline $\begin{array}{l}\text { 8. La presencia de alumnado con limitaciones en la movilidad } \\
\text { en el aula dificulta el rendimiento del alumnado sin } \\
\text { limitaciones. }\end{array}$ & 4,00 & 4,70 \\
\hline
\end{tabular}

Los diferentes elementos propuestos para la dimensión «Planificación didáctica y diseño curricular» son valorados como pertinentes y claros (ver tabla 3 ). De entre todos los ítems aquí recogidos, el elemento que muestra una menor pertinencia es el número 22 , referente al diseño de herramientas de evaluación que cumplan con la función formativa de todo el alumnado. Los demás ítems de la dimensión obtienen una puntuación alta en ambos criterios de valoración. 
Tabla 3. Pertinencia y claridad de la dimensión «Planificación didáctica y diseño curricular»

\begin{tabular}{|c|c|c|c|}
\hline & ELEMENTOS & $\mathbf{P}$ & C \\
\hline 9. & $\begin{array}{l}\text { Conozco cuáles son los objetivos generales del área de Educación } \\
\text { Física. }\end{array}$ & 4,82 & 5,00 \\
\hline 10. & $\begin{array}{l}\text { Conozco las funciones descritas en el currículo para trabajar con } \\
\text { alumnado }\end{array}$ & 4,36 & 4,36 \\
\hline 11. & $\begin{array}{l}\text { Las instalaciones del centro están adaptadas para atender } \\
\text { alumnado con limitaciones en la movilidad dentro del área de } \\
\text { Educación Física. }\end{array}$ & 4,40 & 4,00 \\
\hline 12. & $\begin{array}{l}\text { Dentro de los materiales que dispone el centro hay material } \\
\text { específico para trabajar con alumnado con limitaciones en la } \\
\text { movilidad dentro del área de Educación Física. }\end{array}$ & 4,36 & 4,27 \\
\hline 13. & $\begin{array}{l}\text { Conozco los pasos a seguir para adaptar la programación didáctica } \\
\text { del alumnado con limitaciones en la movilidad dentro del área de } \\
\text { Educación Física. }\end{array}$ & 4,82 & 4,82 \\
\hline & $\begin{array}{l}\text { Recibo ayuda de los especialistas para realizar la adaptación } \\
\text { curricular en mi área. }\end{array}$ & 4,55 & 4,82 \\
\hline 15. & $\begin{array}{l}\text { Las clases están diseñadas con el objetivo de permitir distintos } \\
\text { niveles de realización dentro de una misma sesión de trabajo. }\end{array}$ & 4,40 & 4,80 \\
\hline & $\begin{array}{l}\text { Me considero competente para trabajar con alumnado con } \\
\text { discapacidad motora dentro del área de Educación Física. }\end{array}$ & 4,27 & 4,30 \\
\hline 17. & $\begin{array}{l}\text { Dispongo de las habilidades correspondientes para incentivar que } \\
\text { el alumnado con discapacidad motora se implique en la práctica de } \\
\text { actividad física. }\end{array}$ & 4,55 & 4,82 \\
\hline 18. & $\begin{array}{l}\text { Soy capaz de mantener un ambiente de colaboración en clase } \\
\text { entre alumnos con y sin discapacidad sin que surja ningún tipo de } \\
\text { conflicto entre los mismos. }\end{array}$ & 4,10 & 4,50 \\
\hline 19. & $\begin{array}{l}\text { Me siento competente para coordinarme de una forma eficiente con } \\
\text { otros especialistas de educación especial dentro y fuera del centro. }\end{array}$ & 4,09 & 4,55 \\
\hline 20. & $\begin{array}{l}\text { Me siento competente para tener una relación fluida y cercana con } \\
\text { los padres y madres de los alumnos con discapacidad y mejorar de } \\
\text { esta manera la práctica de actividad física en el área de Educación } \\
\text { Física. }\end{array}$ & 4,40 & 4,30 \\
\hline 21. & $\begin{array}{l}\text { Hago sugerencias al equipo directivo del centro que pueden ser } \\
\text { útiles para lograr una adecuada atención al alumnado con } \\
\text { discapacidad. }\end{array}$ & 4,36 & 4,80 \\
\hline 22. & $\begin{array}{l}\text { Me siento capaz de diseñar herramientas de evaluación que } \\
\text { cumplan con la función formativa de todo el alumnado. }\end{array}$ & 3,91 & 4,30 \\
\hline & $\begin{array}{l}\text { Dispongo de las habilidades necesarias para presentar la tarea con } \\
\text { claridad suficiente para que pueda ser entendida y realizada } \\
\text { correctamente por el alumnado con y sin discapacidad. }\end{array}$ & 4,18 & 4,30 \\
\hline & $\begin{array}{l}\text { Recibo apoyo de la administración educativa para trabajar con el } \\
\text { alumnado con discapacidad motora. }\end{array}$ & 4,09 & 4,36 \\
\hline & $\begin{array}{l}\text { Trabajo de forma coordinada con asociaciones de referencia } \\
\text { especializadas en diferentes tipos de discapacidad. }\end{array}$ & 4,36 & 4,27 \\
\hline & Me gusta trabajar con alumnado con discapacidad. & 4,45 & 4,82 \\
\hline
\end{tabular}

Acercándonos a la dimensión «Necesidades formativas», los catorce elementos que acoge son valorados igualmente como pertinentes y claros (ver tabla 4). 
Tabla 4: Pertinencia y claridad de la dimensión «Necesidades formativas»

27. Los centros educativos ofrecen la formación necesaria para que el profesorado amplié su abanico de conocimientos sobre las discapacidades.

28. Los centros de profesorado ofrecen la formación necesaria para que el profesorado amplié su abanico de conocimientos.

29. Los sindicatos ofrecen la formación necesaria para que el profesorado amplié su abanico de conocimientos.

4,73

$4,45 \quad 4,73$

$4,36 \quad 4,91$

30. La universidad ofrece opciones formativas relacionadas con la atención a la diversidad (Grados, Másteres, cursos de formación, etc).

31. Dispongo de la suficiente formación para dar respuesta al alumnado con discapacidad en las clases de Educación Física.

32. Como profesor procuro actualizarme en temas relacionados con la discapacidad para dar la mejor respuesta al alumnado en el aula.

$4,55 \quad 4,82$

$4,73 \quad 4,91$

$4,70 \quad 4,90$

33. Existe una buena coordinación con el profesorado especialista de educación especial dentro del centro y recibo ayuda de los mismos.

$4,40 \quad 4,80$

34. La formación sobre alumnado con necesidades específicas de apoyo educativo es algo que debe ser de obligado cumplimiento para el profesorado de un centro.

35. Tengo problemas para que el alumnado con discapacidad motora siga el ritmo normal de la clase aun habiendo realizado las pertinentes adaptaciones.

36. Dispongo de monitores de apoyo educativo que me acompañan en el devenir del trabajo con el alumnado con discapacidad.

$4,78 \quad 4,89$

$4,20 \quad 4,80$

$4,30 \quad 4,50$

37. Dispongo de apoyo del equipo de orientación educativa para afrontar mejor las clases de Educación Física con alumnado con discapacidad.

38. Al comenzar el curso académico dispongo de toda la información necesaria (informes, expedientes, etc.) sobre el alumnado con discapacidad.

39. Es necesaria una formación especializada en el trabajo de alumnos con discapacidad en el área de Educación Física.

40. Es necesaria una formación específica sobre discapacidad en todas las áreas del currículo.

$4,10 \quad 4,40$

$4,64 \quad 4,91$

$4,82 \quad 4,73$

$4,64 \quad 4,64$

41. Asisto regularmente a actividades de formación relacionadas con la Educación Física y la discapacidad.

$4,55 \quad 4,91$

Los quince elementos que constituyen la dimensión «Demandas formativas» (ver tabla 5) son claros y pertinentes, según el valor que a ellos han aportado los expertos y las expertas. 
Tabla 5: Pertinencia y claridad de la dimensión "Demandas formativas"

\begin{tabular}{|c|c|c|}
\hline $\begin{array}{l}\text { ELEMENTOS } \\
\end{array}$ & $\mathbf{P}$ & C \\
\hline $\begin{array}{l}\text { 42. Preciso formación específica en el diseño curricular de una } \\
\mathrm{ACl} \text {. }\end{array}$ & 4,55 & 5,00 \\
\hline $\begin{array}{l}\text { 43. Preciso formación específica en la creación y utilización de } \\
\text { materiales y recursos para el área de educación física. }\end{array}$ & 4,64 & 5,00 \\
\hline $\begin{array}{l}\text { 44. Asistiría a cursos de formación especializados en los } \\
\text { diferentes tipos de discapacidad física. }\end{array}$ & 4,73 & 4,82 \\
\hline $\begin{array}{l}\text { 45. Preciso formación específica en técnicas de comunicación } \\
\text { verbal y no verbal con el alumnado. }\end{array}$ & 4,73 & 4,91 \\
\hline $\begin{array}{l}\text { 46. Asistiría a actividades formativas relacionadas con los } \\
\text { sistemas de evaluación del aprendizaje del alumnado con } \\
\text { discapacidad. }\end{array}$ & 4,64 & 4,82 \\
\hline $\begin{array}{l}\text { 47. Considero necesaria una formación centrada en los usos } \\
\text { educativos y didácticos de las tecnologías de la formación y la } \\
\text { comunicación para trabajar con alumnado con discapacidad. }\end{array}$ & 4,73 & 4,64 \\
\hline $\begin{array}{l}\text { 48. Asistiría a cursos en los que se trabajasen actividades físicas } \\
\text { adaptadas para una mejora de la calidad de vida del alumnado } \\
\text { con discapacidad. }\end{array}$ & 4,70 & 4,50 \\
\hline $\begin{array}{l}\text { 49. Es necesaria una formación específica en las } \\
\text { responsabilidades civiles y penales del maestro y maestra. }\end{array}$ & 4,20 & 4,40 \\
\hline $\begin{array}{l}\text { 50. Necesito formarme en contenidos relacionados con los } \\
\text { primeros auxilios en la escuela. }\end{array}$ & 4,89 & 5,00 \\
\hline $\begin{array}{l}\text { 51. Es precisa una formación adecuada en habilidades sociales } \\
\text { para el ejercicio de la docencia. }\end{array}$ & 4,50 & 4,60 \\
\hline 52. Preciso de una formación especializada en psicomotricidad. & 4,70 & 4,80 \\
\hline $\begin{array}{l}\text { 53. Es necesaria una formación especializada en investigación } \\
\text { educativa. }\end{array}$ & 4,30 & 4,50 \\
\hline $\begin{array}{l}\text { 54. Se precisa una formación especializada en el diseño de } \\
\text { proyectos de innovación educativa. }\end{array}$ & 4,30 & 4,50 \\
\hline $\begin{array}{l}\text { 55. Asistiría a cursos de formación relacionados con sistemas de } \\
\text { mediación para la convivencia en el aula. }\end{array}$ & 4,60 & 4,80 \\
\hline $\begin{array}{l}\text { 56. Es necesaria la presencia de cursos en los que se explore la } \\
\text { utilidad de la expresión corporal para el trabajo con alumnado } \\
\text { con discapacidad. }\end{array}$ & 4,60 & 4,60 \\
\hline $\begin{array}{l}\text { 57. Asistiría a cursos de formación relacionados con los juegos } \\
\text { populares y alternativos. }\end{array}$ & 4,60 & 4,80 \\
\hline
\end{tabular}

Al dar a este grupo de profesionales la oportunidad de realizar comentarios abiertos en cada una de las dimensiones planteadas, han argumentado que no todas ellas eran adecuadas para su inclusión en el abanico competencial de la labor profesional de los maestros y maestras de Educación Física, recomendando la reformulación o supresión de algunos elementos así como la inclusión de otros nuevos. Estos pueden describirse del siguiente modo:

- La dimensión «Concepción de la discapacidad en educación primaria y Educación Física», consta de un ítem más que el cuestionario utilizado para evaluar la pertinencia y la claridad del mismo, siendo modificados en su forma de redacción ocho de los elementos propuestos y manteniéndose igual uno.

- En la dimensión «Planificación didáctica y diseño curricular», han sido modificados siete ítems, uno ha sido eliminado y los otros se han respetado tal y como aparecían en el cuestionario de validación y pertinencia. 
- En la dimensión «Necesidades formativas», se han eliminado tres ítems con respecto al cuestionario de validación y pertinencia anteriormente descrito, otros seis se han dejado tal cual aparecían y los seis restantes han sido modificados en su redacción.

- La dimensión «Demandas formativas», consta de tres ítems de nueva creación, siendo uno original eliminado y cinco ítems que fueron modificados en su redacción. El resto se conservan en función del original.

En definitiva, tras la puesta en marcha del juicio de expertos y expertas, el segundo borrador del cuestionario queda configurado tal y como refleja la tabla 6.

Tabla 6. Segundo borrador del cuestionario

\begin{tabular}{lcc}
\hline \multirow{2}{*}{ Dimensiones } & Inicial & Borrador 2 \\
\cline { 2 - 3 } & $\mathbf{N}^{\circ}$ de ítems & $\mathbf{N}^{\circ}$ de ítems \\
\hline - Características del profesorado & 12 & 12 \\
- Concepción de la discapacidad en educación & 8 & 9 \\
$\quad$ primaria y Educación Física & 18 & 17 \\
- Planificación didáctica y diseño curricular & 15 & 12 \\
- Necesidades formativas & 16 & 18 \\
\hline Total & 69 & 68 \\
\hline
\end{tabular}

Una vez analizadas las recomendaciones de los expertos y expertas, pasamos su aplicación experimental a un grupo piloto con la finalidad de identificar si las preguntas son adecuadas, si el enunciado de los ítems es correcto y comprensible, si las preguntas tienen una extensión adecuada, si las respuestas disponen de una categorización correcta, si se produce algún tipo de resistencia o barrera psicológica o rechazo hacia algunas preguntas, si están ordenadas de forma lógica y si la duración de realización del cuestionario está dentro del tiempo aceptable por los encuestados. Procedimos, por tanto, a especificar la fiabilidad y la validez de la medida aportada por el mismo. El grupo ascendió a un total de 35 maestros y maestras de Córdoba capital y provincia, los cuales ejercían en el momento de implementación del cuestionario (curso 2015/2016) su labor docente en el ámbito de la Educación Física. El rango de edad está entre 29-48 años, y todos superan los 5 años de experiencia docente en la rama de Educación Física.

Para el estudio de las garantías científicas del instrumento se han realizado los siguientes análisis:

- Análisis de Consistencia Interna, en el sentido de dotar de significación a los ítems de la prueba, es decir, conseguir que cada uno de ellos mida una porción del rasgo o característica que se desea estudiar (Del Rincón et al., 1995). Para ello utilizamos el coeficiente Alfa de Cronbach.

- Análisis de la capacidad de discriminación de los elementos de modo que se refuerce el carácter unidimensional de la prueba (García, Gil y Rodríguez, 1995). Se utilizó para ello la $t$ de Student entre las medias de los grupos establecidos. 
Realizando una primera aproximación al estudio de la consistencia interna, pudimos advertir cómo los valores correspondientes a cada una de las dimensiones en las que se ha constituido el instrumento indican que las relaciones entre los diferentes elementos de la escala atendiendo a este criterio son muy elevadas (ver tabla 7). Por su parte, el valor total de Alfa en la escala (0.837) indica una correlación alta, un nivel elevado de estabilidad en las respuestas, por lo que el cuestionario presenta indicios de garantías de fiabilidad.

Tabla 7: Coeficientes Alfa en el total del cuestionario en función de la dimensión

\begin{tabular}{lc}
\hline Dimensión & $\begin{array}{c}\text { Coeficiente } \\
\text { Alfa }\end{array}$ \\
\hline Concepción de discapacidad & 0,602 \\
Planificación didáctica y curricular & 0,881 \\
Necesidades formativas & 0,612 \\
Demandas formativas & 0,904 \\
\hline Total & 0,837 \\
\hline
\end{tabular}

Asimismo, el comportamiento de cada uno de los ítems del cuestionario reveló unos coeficientes alfa en todos ellos por encima de 0,830 lo que confirma la afirmación anterior pudiendo concluir que cada uno de los elementos mide una porción del rasgo que deseamos estudiar y, por lo tanto, el instrumento goza de fiabilidad.

Para llevar a cabo el análisis del poder de discriminación de los ítems se han seleccionado se han recodificado en tres grupos en base al valor de la suma total (Bajo, Medio y Alto):

1 = Grupo Bajo (valor mínimo, percentil 33): $(162,189)$

2 = Grupo Medio (percentil 34, percentil 66): $(190,209)$

3 = Grupo Alto (percentil 67, valor máximo): $(210,223)$

La realización de la prueba de $t$ para muestras independientes permitió establecer la existencia o no de diferencia estadística (n.s.=0.05) entre los grupos 1 y 3 . Todos aquellos valores de $p$ menores que 0,05 representan un alto poder de discriminación por parte del ítem. Por su parte, aquellos valores de $p$ iguales o mayores a 0,05 no permiten rechazar la hipótesis nula de igual discriminación y, por tanto, que el ítem discrimine, por lo que éste debe ser revisado. Los resultados obtenidos con esta prueba vienen a expresar que el $82,14 \%$ de los elementos poseen un poder de discriminación estadística aceptable. Cinco ítems fueron modificados al tener como causa una redacción incorrecta. Por otro lado, cinco ítems han sido eliminados porque no cumplían con una discriminación aceptable en el análisis estadístico, y creemos una vez revisados que nos aportan una información diferente a la que queríamos recopilar. Los cambios que ha sufrido el instrumento y que se plasman en la tabla 8 son los siguientes:

- La dimensión «Concepción de la discapacidad en educación primaria y Educación Física» ha eliminado tres elementos procedentes del 
segundo borrador, ha incorporado uno nuevo, ha modificado uno y ha mantenido igual cinco de ellos.

- La dimensión «Planificación didáctica y diseño curricular», mantiene en su origen todos los elementos constitutivos a excepción a la supresión de uno de ellos.

- En la dimensión «Necesidades formativas» se ha eliminado un elemento respecto del borrador pilotado y se ha modificado la redacción de otro de ellos.

- Finalmente, la dimensión «Demandas formativas» consta de los mismos ítems que el cuestionario piloto, salvo un ítem que ha visto modificada su redacción.

Tabla 8: Cuestionario definitivo dirigido al profesorado de Educación Física

\begin{tabular}{llcc}
\hline \multicolumn{1}{c}{ DIMENSIONES } & Borrador 2 & Definitivo \\
\cline { 3 - 4 } & $\mathbf{N}^{\mathbf{0}}$ de ítems & $\mathbf{N}^{\mathbf{0}}$ de ítems \\
\hline 1. & Características del profesorado & 12 & 12 \\
2. & Concepción de la discapacidad en educación & 9 & 8 \\
& primaria y Educación Física & 17 & 16 \\
3. Planificación didáctica y diseño curricular & 12 & 11 \\
4. Necesidades formativas & 18 & 18 \\
5. Demandas formativas & 68 & 65 \\
\hline \multicolumn{2}{l}{ Total }
\end{tabular}

El cuestionario definitivo, de carácter autoadministrado, está configurado por 65 preguntas agrupadas en cinco dimensiones, de los cuáles tres son abiertas, una semicerradas y 58 de elección (53 escalas ordinales, 7 dicotómicas y 1 politómica).

Con el objetivo último de sentar las líneas básicas para el diseño de un plan de formación del profesorado de Educación Física en el trabajo con alumnado con discapacidad física, hemos procedido a llevar a cabo una Análisis Factorial de carácter exploratorio de la dimensión «Demandas formativas». Previo al desarrollo de esta prueba, es imprescindible verificar la adecuación de la técnica a los datos disponibles. Uno de los requisitos que deben cumplirse para la aplicación de esta técnica es que las variables sean concomitantes. En este sentido, conviene estudiar la matriz de correlaciones entre todos los ítems del instrumento con el objetivo de decidir si es apropiado o no someterla a un proceso de factorización. La existencia de correlaciones altas en dicha matriz nos permite deducir la existencia de una interdependencia entre las mismas, suponiendo recomendable el empleo de esta técnica. Su estudio viene determinado por diversos procedimientos estadísticos que, una vez aplicados, velarán por el empleo o no del análisis factorial. Estas técnicas son:

- Identificación del Determinante de la Matriz de Correlaciones: el determinante ha obtenido un valor de $3,148 \cdot 10^{-5}$, extremadamente bajo, lo que indica la existencia de correlaciones altas entre las variables, posibilitando la aplicación de esta técnica.

- Test de esfericidad de Barlett: el valor obtenido es de 478,575 que, con un valor $p<, 01$, ha resultado ser significativo, lo que indica que la matriz 
de correlaciones no es una matriz de identidad, existiendo correlaciones significativas, probablemente altas, dado que el valor hallado en la prueba es estadísticamente alto. Esto indica que la matriz de datos es adecuada para proceder al análisis factorial.

- Correlaciones anti-imagen: un estudio de esta matriz refleja que los coeficientes de correlación, en su mayoría, son menores de ,05, lo que permite realizar el análisis factorial y resumir los 18 ítems en factores.

- Medida de adecuación de la muestra KMO, de Kaiser-Meyer-Olkin: el valor obtenido ha sido de ,683, por lo que es un valor meritorio (en función del baremo para interpretar el índice KMO, Bisquerra, 1989, p. 297), aconsejando la aplicación del análisis factorial.

Como hemos podido apreciar con las pruebas realizadas a partir de la matriz de correlaciones, los datos de que disponemos son aceptables para la aplicación del análisis factorial. A partir de aquí se aplica la técnica extrayendo los componentes principales y sometiéndolos a rotación varimax.

Partiendo de la regla de conservar aquellos componentes cuyos autovalores son mayores que la unidad, obtuvimos un total de 5 factores con una explicación total de la varianza de un $68,086 \%$. El análisis se ha hecho sobre la base de considerar a cada ítem del cuestionario como una variable e integrar a los elementos en los factores obtenidos aquellos que aportan una carga factorial superior a 0,40 (García, Gil y Rodríguez, 2000). Se muestra en la tabla 9 las variables ordenadas para cada factor en cuanto a su correlación con el mismo. 
Tabla 9: Ítems que saturan cada componente como resultado del Análisis Factorial (matriz de componentes rotados)

\begin{tabular}{|c|c|c|c|c|c|}
\hline \multirow{2}{*}{ Elementos } & \multicolumn{5}{|c|}{ Componente } \\
\hline & 1 & 2 & 3 & 4 & 5 \\
\hline $\begin{array}{l}\text { 64. Es necesaria la presencia de cursos en los que se } \\
\text { explore la utilidad de la actividad física y la salud para el }\end{array}$ & ,819 & & & & \\
\hline $\begin{array}{l}\text { trabajo con alumnado con limitaciones en la movilidad } \\
61 \text {. Es necesaria la presencia de cursos en los que se } \\
\text { explore la utilidad de las actividades físicas artístico- } \\
\text { expresivas para el trabajo con alumnado con limitaciones } \\
\text { en la movilidad }\end{array}$ & ,801 & & & & \\
\hline $\begin{array}{l}\text { 62. Es necesaria la presencia de cursos en los que se } \\
\text { explore la utilidad de las habilidades motrices para el } \\
\text { trabajo con alumnado con limitaciones en la movilidad }\end{array}$ & ,738 & & & & \\
\hline $\begin{array}{l}\text { 63. Es necesaria la presencia de cursos en los que se } \\
\text { explore la utilidad de imagen y percepción corporal para } \\
\text { el trabajo con alumnado con limitaciones en la movilidad } \\
65 \text {. Asistiría a cursos de formación relacionados con los }\end{array}$ & ,704 & & & & \\
\hline $\begin{array}{l}\text { juegos populares y alternativos para el trabajo con } \\
\text { alumnado con limitaciones en la movilidad }\end{array}$ &, 586 & & & & \\
\hline $\begin{array}{l}\text { 49. Preciso formación específica en la creación y } \\
\text { utilización de materiales y recursos para el área de } \\
\text { Educación Física }\end{array}$ & & ,872 & & & \\
\hline $\begin{array}{l}\text { 51. Preciso formación específica en técnicas de } \\
\text { comunicación verbal y no verbal y en sistemas } \\
\text { alternativos de comunicación }\end{array}$ & & ,740 & & & \\
\hline $\begin{array}{l}\text { 52. Asistiría a actividades formativas relacionadas con los } \\
\text { sistemas de evaluación del aprendizaje del alumnado con } \\
\text { limitaciones en la movilidad }\end{array}$ & & ,640 & & & \\
\hline $\begin{array}{l}\text { 48. Tengo formación específica en el diseño curricular de } \\
\text { una } \mathrm{ACl}\end{array}$ & & ,506 & & & \\
\hline $\begin{array}{l}\text { 57. Preciso de una formación especializada en } \\
\text { psicomotricidad }\end{array}$ & & & ,821 & & \\
\hline $\begin{array}{l}\text { 59. Se precisa una formación especializada en el diseño } \\
\text { de proyectos de innovación educativa }\end{array}$ & & & ,801 & & \\
\hline $\begin{array}{l}\text { 60. Asistiría a cursos de formación relacionados con } \\
\text { sistemas de mediación para la convivencia en el aula }\end{array}$ & & & ,728 & & \\
\hline $\begin{array}{l}\text { 56. Es precisa una formación adecuada en habilidades } \\
\text { sociales para el ejercicio de la docencia }\end{array}$ & & & & 683 & \\
\hline $\begin{array}{l}\text { 54. Es necesaria una formación específica en las } \\
\text { responsabilidades civiles y penales del maestro y maestra }\end{array}$ & & & & 677 & \\
\hline $\begin{array}{l}\text { 55. Necesito formarme en contenidos relacionados con } \\
\text { los primeros auxilios en la escuela }\end{array}$ & & & & ,647 & \\
\hline $\begin{array}{l}\text { 58. Es necesaria una formación básica en investigación } \\
\text { educativa }\end{array}$ & & & & ,426 & \\
\hline $\begin{array}{l}\text { 50. Asistiría a cursos de formación especializados en } \\
\text { personas con limitaciones en la movilidad }\end{array}$ & & & & & ,867 \\
\hline $\begin{array}{l}\text { 53. Considero necesaria una formación centrada en los } \\
\text { usos educativos y didácticos de las tecnologías de la } \\
\text { información y la comunicación para trabajar con } \\
\text { alumnado con discapacidad }\end{array}$ & & & & & ,778 \\
\hline
\end{tabular}

Los factores que han resultado del análisis aquí realizado así como su aportación al modelo (varianza explicada por cada uno de ellos) y la fiabilidad de los mismos (valores de Alfa de Cronbach), así como su denominación, se muestran en la tabla 10. 
Tabla 10: Factores obtenidos, aportación al modelo de cada componente e índice de fiabilidad de los elementos resultantes del Análisis Factorial

\begin{tabular}{clcc}
\hline Factor & \multicolumn{1}{c}{ Denominación } & $\begin{array}{c}\% \text { de la } \\
\text { varianza }\end{array}$ & $\begin{array}{c}\text { Alfa de } \\
\text { Cronbach }\end{array}$ \\
\hline 1 & Didáctica de la Educación Física & 19,875 & 0,850 \\
\hline 2 & Herramientas didáctico educativas & 13,334 & 0,723 \\
\hline 3 & Estrategias de trabajo en el aula & 12,844 & 0,764 \\
\hline 4 & Habilidades para el ejercicio de la docencia & 11,180 & 0,580 \\
\hline 5 & Recursos TIC & 10,853 & 0,675 \\
\hline
\end{tabular}

Se explican aquí los 5 componentes obtenidos y que son la base para el diseño del plan de formación docente pretendido:

- Didáctica de la Educación Física: Este primer componente, que explica un $19,875 \%$ de la varianza del criterio, recoge aspectos relacionados con los cuatro bloques de contenido que la legislación LOMCE incluye en su Real Decreto 126/2014, el cual establece el currículo en las enseñanzas mínimas de la educación primaria y que se concreta en su Decreto 97/2015 y en su Orden 17 de marzo de 2015 en Andalucía. Los bloques de contenido que se hacen referencia y que son demandados para la formación por los maestros y maestras de Educación Física encuestados están relacionados con la actividad física y salud, las actividades físicas artístico-expresivas, las habilidades motrices, los juegos populares y alternativos y los deportes.

- Herramientas didáctico educativas: El segundo componente que nos encontramos, que explica un $13,334 \%$ de la varianza, engloba aspectos en los cuales los y las profesionales del área requieren formación en algunas herramientas para afrontar de la mejor forma posible sus clases de Educación Física, y para ello demandan formación en la creación y utilización de materiales para el trabajo en el área de Educación Física con alumnado con discapacidad motora, técnicas de comunicación verbal y no verbal y en sistemas alternativos de comunicación $\mathrm{y}$, por último, en la utilización y conocimiento de diferentes sistemas de evaluación, ya que la legislación vigente propone el elemento curricular de criterios de evaluación junto a sus estándares de aprendizaje e indicadores como los elementos a partir de los cuales parten los diferentes elementos de la programación para poder conseguir, al final de la etapa educativa, el mayor grado de adquisición de las competencias clave y los objetivos generales de la etapa (Rementería, 2009).

- Estrategias de trabajo en el aula: Este tercer componente recoge aspectos de vital importancia para que el trabajo en el aula con alumnado con discapacidad motora sea lo más eficaz y eficiente posible para todo el alumnado. Basándonos en el principio de equidad que tanto buscan las legislaciones vigentes y en la Orden de 25 de julio de 2008, por la que se regula la atención a la diversidad del alumnado que cursa la educación básica en los centros docentes públicos de Andalucía, nos encontramos con la demanda de una formación específica en psicomotricidad por la importancia que tiene este tipo de trabajo con alumnado con discapacidad; se precisa también de formación en el diseño de proyectos de innovación para la 
actualización en la materia, aprendizaje de trabajo en grupo y de sistemas de mediación en conflictos.

- Habilidades sociales para el ejercicio de la docencia: El siguiente componente al que se hace referencia aborda formación en habilidades sociales para el ejercicio de la docencia, en responsabilidades civiles y penales como docente de educación primaria y, más en concreto, como docente de Educación Física, y en un tema de una alta importancia en la actualidad como son los primeros auxilios. Además de estos contenidos y demandas formativas, en este apartado se reclama formación en investigación educativa como apoyo a la innovación docente.

- Recursos TIC: El último componente encontrado engloba aspectos relacionados con el uso de las nuevas tecnologías de la información y comunicación y aspectos relacionados con la competencia digital, empleando los términos de Prats, Camerino y Coiduras (2013). En este aspecto, se requiere formación en la creación de recursos tecnológicos y la utilización de recursos y materiales relacionados con la competencia digital, tanto a nivel general como específica, más concretamente en recursos digitales que faciliten el trabajo en el aula con alumnado con limitaciones en la movilidad.

\section{CONCLUSIONES}

Las diferentes pruebas realizadas para valorar la fiabilidad y la validez del cuestionario diseñado al efecto dirigido al profesorado de Educación Primaria especialista en Educación Física, cuya finalidad es describir la realidad del trabajo de aula cuando en su acción didáctica participa alumnado con discapacidad física, y revelar las carencias de formación al respecto, permiten afirmar que goza de las suficientes garantías científicas de fiabilidad y validez para ser aplicado, analizado e interpretado. Este se ha configurado como un protocolo formado por ítems de valoración escalar bajo cuatro dimensiones de análisis: concepción de la discapacidad, planificación didáctica y diseño curricular, necesidades formativas y demandas formativas.

El éxito del cuestionario ha dependido, en gran medida, del grado de adecuación del contenido de las preguntas al tema objeto de la investigación, del grado de comprensión por parte del profesorado encuestado del contenido que está leyendo y del ajuste de las respuestas obtenidas a dicho contenido.

Asimismo, se han construido gracias a su aplicación experimental, las dimensiones formativas a partir de las que se generarán las diferentes acciones formativas que apoyen la labor docente cuando se enfrenta en el aula al trabajo diario con alumnado con discapacidad física y que son: didáctica de la Educación Física, herramientas didáctico-educativas, estrategias de trabajo en el aula, habilidades para el ejercicio de la docencia y recursos TIC. Hay que señalar que Calderón y Martínez de Ojeda (2014) ya hicieron referencia a las citadas dimensiones dentro de los planes de formación permanente del profesorado de Educación Física, lo que contribuye a dar más valor pragmático al instrumento aquí ofrecido. 


\section{REFERENCIAS BIBLIOGRÁFICAS}

1. Arnaiz, P.; De Haro, R. y Guirao, J.M. (2015). La evaluación en educación primaria como punto de partida para el desarrollo de planes de mejora inclusivos en la región de Murcia. Revista electrónica interuniversitaria de formación del profesorado, 18 (1), 103-122.

2. Beltrán, B. (2008). Guía para la atención educativa a los alumnos y alumnas con discapacidad motora. VI Encuentro de Diversired. 5- 36 Centro del profesorado de Alcalá de Guadaira. Junta de Andalucía.

3. Bisquerra, R. (1989). Métodos de investigación educativa. Guía práctica. Barcelona: Ceac.

4. Calderón, A. y Martínez de Ojeda, D. (2014). La formación permanente del profesorado de Educación Física. Propuesta de enseñanza del modelo de Educación Deportiva. Revista de Educación, 363, 128-153.

5. Caus, N.; Santos, E.; Blasco, J. y Vega, L. (2013). Procedimiento de actuación ante la inclusión de alumnado con discapacidad en el área de Educación Física. Apunts. Educación Física y Deportes, 112, 37-45.

6. Decreto $97 / 2015$, de 3 de marzo, por el que se establece la ordenación y el currículo de educación primaria en la Comunidad Autónoma de Andalucía (Boletín Oficial de Estado número 50, de 13 de marzo de 2015).

7. Del Rincón, D. et al. (1995). Técnicas de investigación en Ciencias Sociales. Madrid: Dikinson.

8. Díaz, E. (2010). Ciudadanía, identidad y exclusión social de las personas con discapacidad. Política y Sociedad, 47 (1),115-135

9. Echeita, G. y Ainscow, M. (2011). La Educación inclusiva como derecho. Marco de referencia y pautas de acción para el desarrollo de una revolución pendiente. Madrid: UAM.

10. Egea, C. y Sarabia, A. (2014). Experiencias de aplicación en España de la Clasificación Internacional de Deficiencias, Discapacidades y Minusvalías. Madrid: Real patronato sobre discapacidad.

11. García, E.; Gil, J. y Rodríguez, G. (1995). Introducción a la teoría clásica de los tests. Sevilla: Grupodelta.

12. García, E.; Gil, J. y Rodríguez, G. (2000). Análisis factorial, cuadernos de estadística. Madrid. La Muralla.

13. Jenaro, C. et al. (2014). Necesidades educativas e inclusión escolar: el peso de las actitudes. International Journal of Developmental and Educational Psychology, 4 (1), 605-612.

14. Junta de Andalucía (2014). Orden de 31 de julio de 2014, por la que se aprueba el III Plan Andaluz de Formación Permanente del Profesorado (Boletín Oficial de la Junta de Andalucía numero 170, de 2 de agosto de 2014).

15. Junta de Andalucía (2015). Instrucciones 22 de junio de 2015, de la Dirección General de Participación y Equidad, por las que se establece el protocolo de detección, identificación del alumnado con necesidades especificas de apoyo educativo y organización de la respuesta educativa.

Recuperado

de

http://www.juntadeandalucia.es/educacion/webportal/web/inspeccion/noticias//contenidos/detalle/instrucciones-de-22-de-junio-de-2015-de-la-direccion-general-departicipacion-y-equidad-por-las-2

16. Orden de 25 de julio de 2008 , por la que se regula la atención a la diversidad del alumnado que cursa la educación básica en los centros docentes públicos de Andalucía (Boletín Oficial de la Junta de Andalucía número 167, de 22 de agosto de 2008).

17. Ley Orgánica $8 / 2013$, de 9 de Diciembre, para la mejora de la calidad educativa (Boletín Oficial de Estado número 295, de 10 de diciembre de 2013).

18. Margalef, L. (2000). ¿De qué estamos hablando cuando nos referimos a la educación en y para la diversidad? En M. Martín y L. Margalef (Coords.), La educación para la diversidad: múltiples miradas (pp. 16-41). Alcalá: Universidad de Alcalá. 
19. Muntaner, J.J. (2000). La igualdad de oportunidades en la escuela de la diversidad. Palma de Mallorca: Universitat de les Illes Ballears.

20. Muntaner, J.J. (2010). De la integración a la inclusión: un nuevo modelo educativo. En P. Arnáiz, P.; M.D. Hurtado y F.J. Soto (Coords.), 25 años de integración escolar en España: Tecnología e Inclusión en el ámbito educativo, laboral y comunitario (pp. 2-24). Murcia: Consejería de Educación, Formación y Empleo.

21. Muñoz, S. (2014). La interpretación estatutaria del sistema constitucional de distribución de competencias. Recuperado de: http://www.memoriadigitalvasca.es/handle/10357/19592

22. Orden de 17 de marzo de 2015, por la que se desarrolla el currículo correspondiente a la Educación Primaria en Andalucía (Boletín Oficial de la Junta de Andalucía número 60, de 27 de marzo de 2015).

23. Pantoja, A. y Montijano, J. (2014). Estudio sobre hábitos de actividad física saludable en niños de educación primaria de Jaén capital. Apunts, 107, 13-23.

24. Penagos, N.A. (2013). Concepciones y Prácticas de los docentes de Educación Física frente a la inclusión de los niños con necesidades educativas especiales en los colegios oficiales de la Localidad Barrios Unidos. Bogotá: Universidad de la Sabana.

25. Prats, Q.; Camerino, O. y Coiduras, J.L. (2013). Introducción de las TIC en Educación Física. Estudio descriptivo sobre la situación actual. Apunts, 113, 37-44.

26. Real Decreto 126/2014, de 28 de febrero, por el que se establece el currículo básico de la Educación Primaria (Boletín Oficial de Estado número 52, de 1 de marzo de 2014).

27. Rementería, C.J. (2009) La bitácora del alumno en Educación Física. EFDdeportes.com, 139. Recuperado de http://www.efdeportes.com/efd139/la-bitacora-del-alumno-eneducacion-fisica.htm.

28. Ríos, L.A. y Martínez, C. (2004). El bádminton adaptado: Una propuesta para la integración de alumnos con discapacidad física. Badajoz: Universidad de Extremadura.

29. Ríos, M. (2007). Manual de Educación Física adaptada al alumnado con discapacidad. Barcelona: Paidotribo.

30. Ríos, M. (2009). La inclusión en el área de Educación Física en España. Análisis de las barreras para la participación y aprendizaje. Ágora para la EF y el Deporte, 9, 83-114.

31. Ruiz, C. (2002). Instrumentos de Investigación Educativa. Caracas: Fedupel.

32. Velázquez, C.; Aranda, A. F., y Pastor, V. M. L. (2014). Aprendizaje cooperativo en Educación Física. Movimento: revista da Escola de Educação Física, 1 (20), 239-259.

Referencias totales citadas: 32

Referencias citadas correspondientes a la Rev Ib CC Act Fis Dep: 0 